\title{
Report on USDA Ultraviolet Spectroradiometers
}

\section{E. A. Early \\ Ambler Thompson}

U.S. DEPARTMENT OF COMMERCE Technology Administration National Institute of Standards and Technology Optical Technology Division

Gaithersburg, MD 20899

QC

100

.056

N0.5871

1996 



\title{
NISTIR 5871
}

\section{Report on USDA Ultraviolet Spectroradiometers}

\author{
E. A. Early \\ Ambler Thompson
}

U.S. DEPARTMENT OF COMMERCE Technology Administration National Institute of Standards and Technology

Optical Technology Division Gaithersburg, MD 20899

July 1996

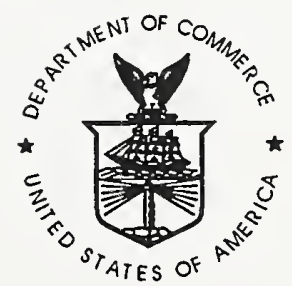

U.S. DEPARTMENT OF COMMERCE Michael Kantor, Secretary

TECHNOLOGY ADMINISTRATION

Mary L. Good, Under Secretary for Technology

NATIONAL INSTITUTE OF STANDARDS

AND TECHNOLOGY

Arati Prabhakar, Director 



\title{
REPORT ON USDA ULTRAVIOLET SPECTRORADIOMETERS
}

\author{
E. A. Early and Ambler Thompson \\ Optical Technology Division \\ National Institute of Standards and Technology \\ Gaithersburg, MD 20899
}

\begin{abstract}
Two ultraviolet spectroradiometers manufactured for the U.S. Department of Agriculture monitoring network were characterized. From measurements of the linearity of the instruments, the dead time of the photon counting system was approximately $12 \mathrm{~ns}$. From spectral scans of the emission lines of a Hg lamp, the wavelength repeatability of the instruments is $0.02 \mathrm{~nm}$, while the expanded uncertainty is $0.04 \mathrm{~nm}$. Spectral scans of the $325 \mathrm{~nm}$ line of a HeCd laser showed that the full-width-at-half-maximum bandwidth of the instruments is $0.3 \mathrm{~nm}$ and that the monochromators have a stray-light rejection of $10^{-8}$. The spectral irradiance responsivity, determined with FEL-type irradiance standard lamps, changes both with movement of the instruments and with time.
\end{abstract}




\section{DESCRIPTION OF THE INSTRUMENTS}

Two ultraviolet spectroradiometers manufactured by Research Support Instruments, Inc. (RSI) ${ }^{\dagger}$ and the Atmospheric Science Research Center (ASRC) at SUNY, Albany were characterized. The instruments were designed for long-term field monitoring of the solar ultraviolet irradiance at the surface of the Earth, and were designated Unit 901 and Unit 902. Each instrument consists of a cylindrical housing containing the foreoptic, monochromator, and detector, and a separate box containing the power supply and interface to a remote computer. When deployed in the field, each instrument also had associated nitrogen purge and cooling systems. The instruments can be mounted either horizontally or vertically, the latter being the orientation for operation in the field.

The receiving aperture of the foreoptic is a Spectralon disk with a diameter of $2.54 \mathrm{~cm}$ and shaped to closely approximate a Lambertian collector. A Spectralon integrating cavity behind the disk collects the transmitted radiation, and the exit port of this cavity is imaged onto the entrance slit of the monochromator. A Hg emission lamp placed outside the cavity and near the optic axis is used for wavelength calibration of the instrument.

The monochromator is a folded double $1 / 8 \mathrm{~m}$ Ebert manufactured by RSI. The wavelength setting of the monochromator is determined by the angle of the diffraction grating, which in turn is set by a rod connected to a nut that travels along a lead screw. A rub bar attached to the top of the nut prevents the nut from rotating about the screw. The screw is turned by a reducer connected to a stepping motor, and a chain attached to the nut wraps around two pulleys, one of which turns the shaft of an absolute encoder. Therefore, the wavelength setting can be determined absolutely either by the encoder or by the number of motor steps, if the steps are counted from a known starting position. The nominal wavelength range of the instruments is $280 \mathrm{~nm}$ to $400 \mathrm{~nm}$.

The detector is a Hamamatsu R2371HA photomultiplier tube (PMT) maintained at $15^{\circ} \mathrm{C}$ by a Peltier cooler. The number of pulses from the tube resulting from detected photons is divided by five before being counted.

The instrument communicates with an external computer over an RS-232 serial line. A microprocessor interprets commands given to the instrument into the appropriate action(s). For example, the command HG-ON turns on the internal Hg lamp. The most common measurement is a spectral scan, which consists of setting the wavelength to the appropriate initial value and accumulating photon counts for a specified time, and then repeatedly advancing a given number of motor steps, stopping, and accumulating photon counts until the final wavelength is reached. All spectral scans are performed with increasing wavelength. When the wavelength was decreased, for example at the completion of a spectral scan, the diffraction grating angle in terms of motor steps was

\footnotetext{
${ }^{\dagger}$ Certain commercial equipment, instruments, or materials are identified in this paper in order to specify the experimental procedure adequately. Such identification is not intended to imply recommendation or endorsement by the National Institute of Standards and Technology, nor is it intended to imply that the materials or equipment identified are necessarily the best available for the purpose.
} 
intentionally overshot by 500 steps and then increased to the desired value to remove any backlash. 


\section{OVERVIEW OF MEASUREMENTS}

The guiding principle in evaluating the instrument is to isolate and characterize as many parameters as practical that affect its overall performance. In other words, by evaluating each separate component or parameter of the instrument, its overall performance can be understood. The basis for performing this type of evaluation is the simplified measurement equation [1], given by

$$
S\left(\lambda_{0}\right)=\int E(\lambda) R\left(\lambda_{0}, \lambda\right) d \lambda
$$

where $\lambda[\mathrm{nm}]$ is the wavelength, $\lambda_{0}[\mathrm{~nm}]$ is the wavelength setting of the monochromator, $S\left(\lambda_{0}\right)$ [Counts s $\left.{ }^{-1}\right]$ is the output signal of the photon counting circuit, $E(\lambda)\left[\mathrm{mW} \mathrm{m}^{-2} \mathrm{~nm}^{-1}\right]$ is the spectral irradiance of the source, and $R\left(\lambda_{0}, \lambda\right)\left[\left(\right.\right.$ Counts s-1) / $\left.\left(\mathrm{mW} \mathrm{m}^{-2} \mathrm{~nm}^{-1}\right)\right]$ is the spectral irradiance responsivity function of the instrument. Further, the spectral irradiance responsivity function is given by

$$
R\left(\lambda_{0}, \lambda\right)=R(\lambda) z\left(\lambda-\lambda_{0}\right),
$$

where $R(\lambda)\left[\left(\right.\right.$ Counts s$\left.\left.^{-1}\right) /\left(\mathrm{mW} \mathrm{m}^{-2}\right)\right]$ is the irradiance response function and $z\left(\lambda-\lambda_{0}\right)$ is the dimensionless slit-scattering function. Combining Eqs. (2.1) and (2.2) yields

$$
S\left(\lambda_{0}\right)=\int E(\lambda) R(\lambda) z\left(\lambda-\lambda_{0}\right) d \lambda
$$

The spectral irradiance can either be a known quantity, as with a standard lamp, or be the desired result, as with measurements in the ultraviolet region of the solar spectrum. The response function varies slowly with wavelength, and indicates the sensitivity of the instrument to light at a given wavelength. Conversely, the slit-scattering function is a rapidly varying function of wavelength, ideally independent of $\lambda_{0}$ and triangular in shape, and indicates the bandwidth $\Delta \lambda$ of the instrument.

The purpose of each experiment detailed in the following sections is to separately characterize various parameters in Eq. (2.3). Ideally, the signal $S\left(\lambda_{0}\right)$ is directly proportional to the power $\phi$ incident on the receiving aperture over a wide range of powers. In practice, there are deviations from linearity, which were experimentally determined by the beam addition method and described in Section III.

The wavelength repeatability and uncertainty of the monochromator are very important in measurements of solar ultraviolet irradiance, where a $0.02 \mathrm{~nm}$ wavelength uncertainty at $295 \mathrm{~nm}$ results in a $2 \%$ irradiance relative uncertainty. To determine the wavelength of the monochromator, sources are used with emission lines at known wavelengths within the range of the instrument. These were $\mathrm{Hg}$ and $\mathrm{Cd}$ emission lamps and a $\mathrm{HeCd}$ laser, and results from wavelength scans of these sources are given in Section IV. 
The slit-scattering function was determined by the experiments described in Section V. With a monochromatic source operating at wavelength $\lambda^{\prime}$, the source irradiance reduces to $E(\lambda)=E_{0} \delta\left(\lambda-\lambda^{\prime}\right)$, and Eq. (2.3) becomes

$$
S\left(\lambda_{0}\right)=E_{0} R\left(\lambda^{\prime}\right) z\left(\lambda^{\prime}-\lambda_{0}\right) \text {. }
$$

A HeCd laser was used as the monochromatic source because of the relatively high power in the beam. The power is also important when determining the stray-light rejection of the instrument, defined as the value of $z\left(\lambda-\lambda_{0}\right)$ at wavelengths far from $\lambda_{0}$.

Given a source with a known spectral irradiance $E_{\mathrm{s}}(\lambda)$, and assuming that $E_{\mathrm{S}}(\lambda)$ and $R(\lambda)$ vary slowly over the wavelength range for which $z\left(\lambda-\lambda_{0}\right)$ is appreciable, Eq. (2.3) becomes

$$
S\left(\lambda_{0}\right)=E_{s}\left(\lambda_{0}\right) R\left(\lambda_{0}\right) \int z\left(\lambda-\lambda_{0}\right) d \lambda .
$$

The product $R\left(\lambda_{0}\right) \int z\left(\lambda-\lambda_{0}\right) d \lambda\left[\left(\right.\right.$ Counts $\left.\left.\mathrm{s}^{-1}\right) /\left(\mathrm{mW} \mathrm{m}^{-2} \mathrm{~nm}^{-1}\right)\right]$ is the spectral irradiance responsivity, and reduces to $R\left(\lambda_{0}\right) \Delta \lambda$ for an ideal slit-scattering function. An FEL-type irradiance standard lamp was used to determine the spectral irradiance responsivity of the instrument, described in Section VI.

Finally, results from noise measurements are presented in Section VII, where the signal is recorded multiple times at a fixed wavelength, and conclusions are given in Section VIII. All expanded uncertainties in this report are expressed with a coverage factor $k=2$, which provides a level of confidence of about $95 \%$ [2].

The results presented here were obtained from characterizations performed on both instruments. Unit 901 was characterized at NIST from January 17 to April 27, 1995, while both instruments were partially characterized at the North American Interagency Ultraviolet Monitoring Spectroradiometer Intercomparisons held outside Boulder, Colorado, Unit 902 during the intercomparison from September 19 to 29, 1994 and Unit 901 during the intercomparison from June 14 to 22, 1995. Two changes were made in Unit 901 between its characterizations at the National Institute of Standards and Technology (NIST) and those at the Boulder intercomparison. The PMT was changed to a factory-selected Hamamatsu R2371PHA and the pulse amplification and discriminator circuits of the photon counting system were improved. 


\section{LINEARITY}

The linearity of the instrument was measured by the beam addition method, in which a light beam from a single source is split into two paths, variably attenuated by neutral density filters, recombined and passed through a third filter, and detected by the instrument. The power through the third filter $\phi$, which is that detected by the instrument, is given by

$$
\phi=\phi_{1}+\phi_{2}
$$

where $\phi_{1}$ is the power along one path and $\phi_{2}$ is the power along the other path. By using different combinations of filters, the incident power on the receiving aperture of the instrument can be varied to cover a dynamic range of approximately 3000 to one. The instrument which performs this measurement, Beamcon III, was specifically designed to characterize detectors at low power levels, approaching the noise-equivalent-power of the detector, and is described in more detail elsewhere [3].

In a photon counting circuit, nonlinearity is caused by the dead time, defined as the minimum time between photoelectrons necessary for proper counting of all pulses. For a thermal source, the measured signal $S^{\prime}$ is given by

$$
S^{\prime}=S /\left(1+t_{\mathrm{d}} S\right)
$$

where $S$ is the actual signal and $t_{\mathrm{d}}$ is the dead time. This can be easily solved for $S$, with the result

$$
S=S^{\prime} /\left(1-t_{\mathrm{d}} S^{\prime}\right)
$$

The signal $S$ given by Eq. (3.2) is also directly proportional to the power $\phi$ in Eq. (3.1). Therefore, a least-squares technique is used to solve for the powers $\phi_{1}$ and $\phi_{2}$ as well as for the dead time $t_{\mathrm{d}}$.

The linearity of Unit 901 was determined in this manner from five experimental runs on February 8, 1995 and from four runs on February 9, 1995. The source was a Xe lamp and the wavelength of the monochromator was approximately $398 \mathrm{~nm}$. This resulted in a maximum signal of 425,000 counts/s. The measured count rate was converted to photons/s by multiplying by five before performing the calculation of the dead time. The average dead time from these nine runs was $12.3 \mathrm{~ns}$ with a standard deviation of the mean of $1.2 \mathrm{~ns}$. A plot of the measured signal divided by the calculated power as a function of measured signal for one of the runs is given in Fig. 3.1. At a count of $2^{20}$, the maximum permitted by the photon counting system, the dead-time corrected signal is greater than the measured signal by $6.4 \%$. 


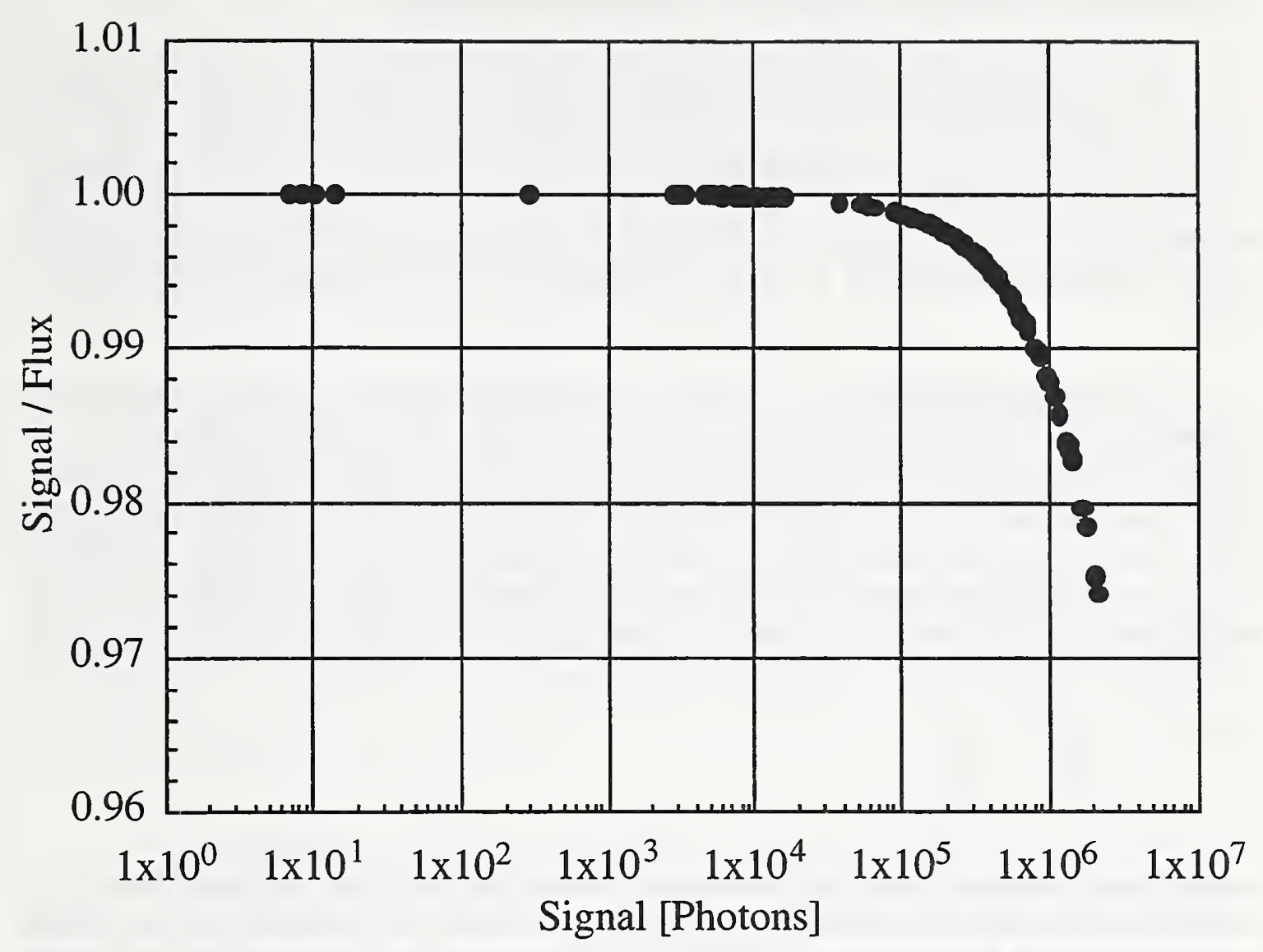

Figure 3.1. Measured signal divided by calculated power as a function of measured signal for the photon counting circuit of Unit 901. 


\section{WAVELENGTH REPEATABILITY AND UNCERTAINTY}

The wavelength of the monochromator in terms of either encoder units or motor steps is determined from the wavelengths of emission lines of the internal $\mathrm{Hg}$ lamp. In typical operation, spectral scans are performed of the emission lines at $296.728 \mathrm{~nm}$ and $334.149 \mathrm{~nm}$ since these are intense singlet lines that span the wavelength range of the monochromator. A linear fit of the centroids of these lines as a function of encoder units and motor steps determines the wavelength calibration of the monochromator in these two units.

Numerous spectral scans of the nine resolvable $\mathrm{Hg}$ emission lines of the internal lamp at wavelengths between $280 \mathrm{~nm}$ and $400 \mathrm{~nm}$ were performed on Unit 901 from January 18, 1995 to April 25, 1995. These nine lines occur at $289.360 \mathrm{~nm}, 292.541 \mathrm{~nm}$, $296.728 \mathrm{~nm}, 302.197 \mathrm{~nm}, 312.567 \mathrm{~nm}, 313.173 \mathrm{~nm}, 334.149 \mathrm{~nm}, 365.016 \mathrm{~nm}$, and $366.323 \mathrm{~nm}$, with only the fourth and sixth lines being unresolved multiplets. Of these scans, eleven were performed with two motor steps between each wavelength and a $2 \mathrm{~s}$ integration time. The centroids of each emission line for each scan were determined from the formula

$$
C=\sum_{i=1}^{n} S_{i} U_{i} / \sum_{i=1}^{n} S_{i},
$$

where $C$ is the centroid and $i$ indexes those wavelength units and signals, $U_{\mathrm{i}}$ and $S_{\mathrm{i}}$, respectively, for which the signal is greater than $1 / 4$ times the maximum signal. This simple method for determining the centroid agrees within several encoder units of values determined using more sophisticated techniques. The centroid of the $296.728 \mathrm{~nm}$ emission line was set to 5000 motor steps, and the centroids of the other lines in terms of motor steps were adjusted accordingly. The wavelength calibration for each scan was determined using the procedure described in the preceding paragraph. The average of the fits is

$$
\begin{aligned}
& \lambda=\left(2.67886 \times 10^{-3} \mathrm{~nm}\right)^{*}(\text { encoder units })+238.66 \mathrm{~nm} \text { and } \\
& \lambda=\left(4.08422 \times 10^{-3} \mathrm{~nm}\right) *(\text { motor steps })+276.31 \mathrm{~nm},
\end{aligned}
$$

where $\lambda$ is the wavelength of the monochromator.

Unfortunately, the encoder units did not smoothly track the motor steps. This is illustrated in Fig. 4.1, where the signal from a wavelength scan of the $296.728 \mathrm{~nm}$ emission line is plotted as a function of (a) encoder units and (b) motor steps. The tracking problem is apparent from comparing the smooth curve in Fig. 4.1(b) with the "jagged" curve in Fig. 4.1(a). The problem can be so severe that the encoder unit decreases after a positive motor step. The most likely cause of this problem is the mechanical linkage between the nut on the lead screw and the encoder shaft. The nut can twist and relax slightly about three axes, causing a "stick-slip" behavior in the encoder from these undesired torsions. 
The wavelength repeatability of the monochromator is given by the standard deviation of the centroids of the nine $\mathrm{Hg}$ emission lines determined by the eleven scans. The results are shown in Fig. 4.2, where the standard deviation for each line is plotted as a function of wavelength for both encoder units and motor steps. The repeatability in terms of motor steps is $0.02 \mathrm{~nm}$ or less, with the best repeatability at $296.728 \mathrm{~nm}$ since the motor steps were adjusted to the centroid at that wavelength. In terms of encoder units, however, the repeatability may be as great as $0.04 \mathrm{~nm}$, a two-fold increase over the repeatability obtained with the motor steps. This increase is likely a manifestation of the tracking problem of the encoder detailed in the preceding paragraph.

The uncertainty of the wavelength calibration is determined by the difference between the centroids calculated from the wavelength calibration and those calculated from the scans of emission lines. For the eleven wavelength scans of the internal $\mathrm{Hg}$ lamp performed on Unit 901 at NIST, the wavelength calibration is given by Eqs. (4.1) and (4.2). The average differences, in units of wavelength, between the two calculations of the centroids are shown in Fig. 4.3 as a function of wavelength for both encoder units and motor steps. The differences are zero at $296.728 \mathrm{~nm}$ and $334.149 \mathrm{~nm}$ since these two wavelengths are used in the linear fit of centroid as a function of wavelength. At the other wavelengths, the difference is less than $0.04 \mathrm{~nm}$, with the encoder units performing slightly better than the motor steps.

The wavelength uncertainty of Unit 901 was also determined at the Boulder intercomparison from seven wavelength scans of external $\mathrm{Hg}$ and $\mathrm{Cd}$ emission lamps and a $\mathrm{HeCd}$ laser. The $\mathrm{Cd}$ lamp has emission lines within the wavelength range of the instrument at $288.086 \mathrm{~nm}, 298.079 \mathrm{~nm}, 308.122 \mathrm{~nm}, 313.317 \mathrm{~nm}, 326.106 \mathrm{~nm}, 340.365 \mathrm{~nm}$, and $346.685 \mathrm{~nm}$, with only the fourth and sixth lines being singlets. The HeCd laser has a single line at $325.029 \mathrm{~nm}$. The average centroids from scans of the internal $\mathrm{Hg}$ lamp on June 18 and June 19 were used to determine the wavelength calibration in terms of both encoder units and motor steps. The centroid of the $296.728 \mathrm{~nm}$ line was adjusted to 3800 motor steps. There was very little change from the calibration obtained at NIST given by Eqs. (4.1) and (4.2). Spectral scans of the external sources were performed on June 15 and June 19, and the centroids were calculated for all sources in terms of encoder units and for only the Hg lamp in terms of motor steps. The differences between the centroids calculated from the wavelength calibration and from the wavelength scans as a function of wavelength are shown in Fig. 4.4(a) for both encoder units and motor steps on both days. As at NIST, the differences in terms of motor steps were less than $0.04 \mathrm{~nm}$ on both days. The expanded uncertainty of the wavelength calibration in terms of encoder units was $0.06 \mathrm{~nm}$ on June 19, which was slightly worse than the motor steps. On June 15 , however, the encoder uncertainty is much worse, especially at the shorter wavelengths. This is probably because the scans on June 15 were performed indoors, while the other scans, including those used to determine the wavelength calibration, were performed outdoors. At all wavelengths, except $325.029 \mathrm{~nm}$, the uncertainty of the encoder decreased from June 15 to June 19. This also illustrates the excursions in wavelength uncertainty that can occur. The differences shown in Fig. 4.3 are less than those shown in Fig. 4.4(a) because the former are averages over many spectral scans, while the latter are from a single spectral scan. 
A similar determination of the wavelength uncertainty of Unit 902 was performed at the Boulder intercomparison. The wavelength calibration of the monochromator was supplied by spectral scans of the internal $\mathrm{Hg}$ lamp, and spectral scans were performed of an external $\mathrm{Hg}$ emission lamp and of a HeCd laser. The differences between the fit and actual centroids of the lines are shown in Fig. 4.4(b) as a function of wavelength for both encoder units and motor steps. The differences for both are less than $0.04 \mathrm{~nm}$, except for the encoder near $365 \mathrm{~nm}$. 

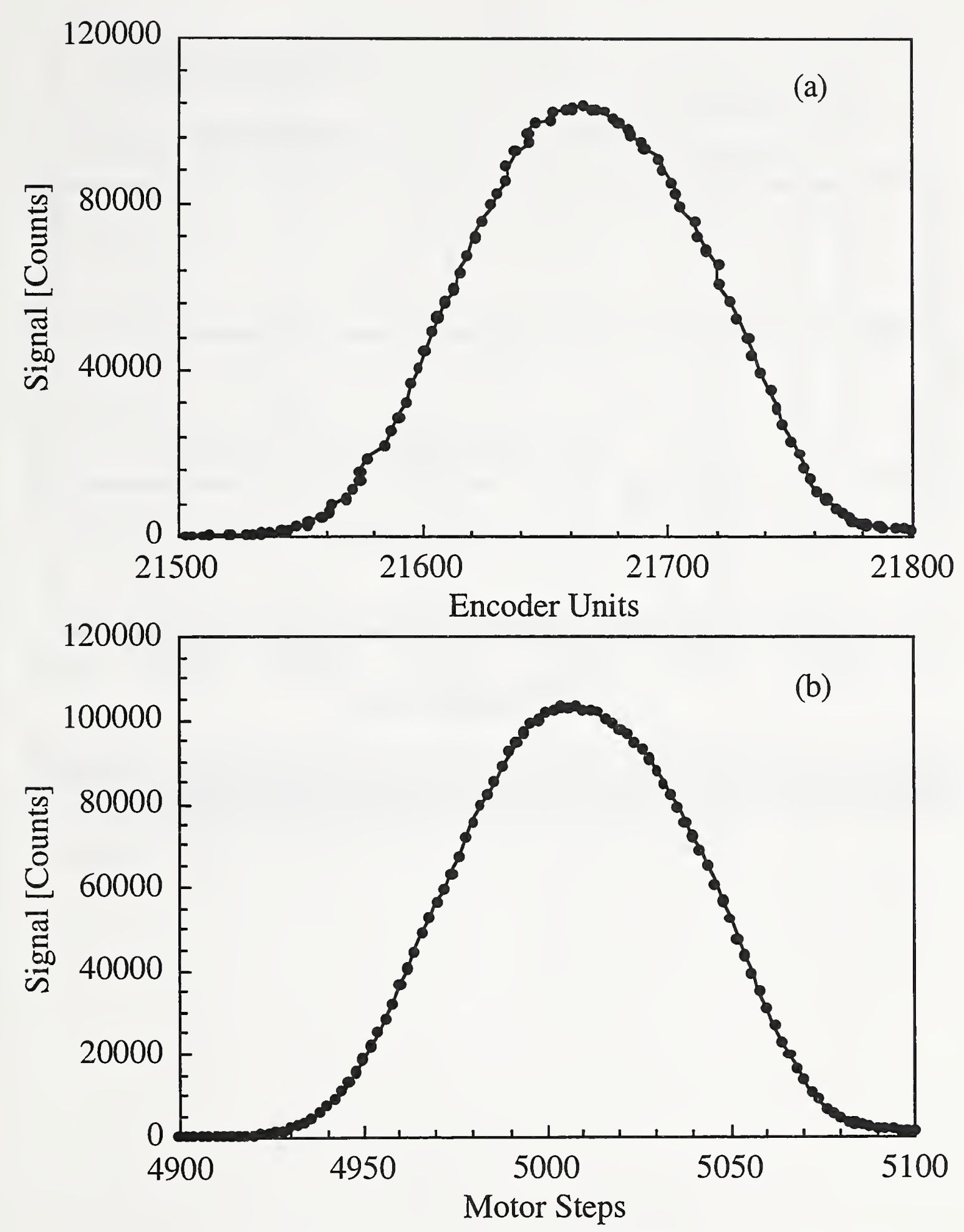

Figure 4.1. Signal as a function of (a) encoder units and (b) motor steps of the $296.728 \mathrm{~nm}$ emission line of $\mathrm{Hg}$ for Unit 901. 


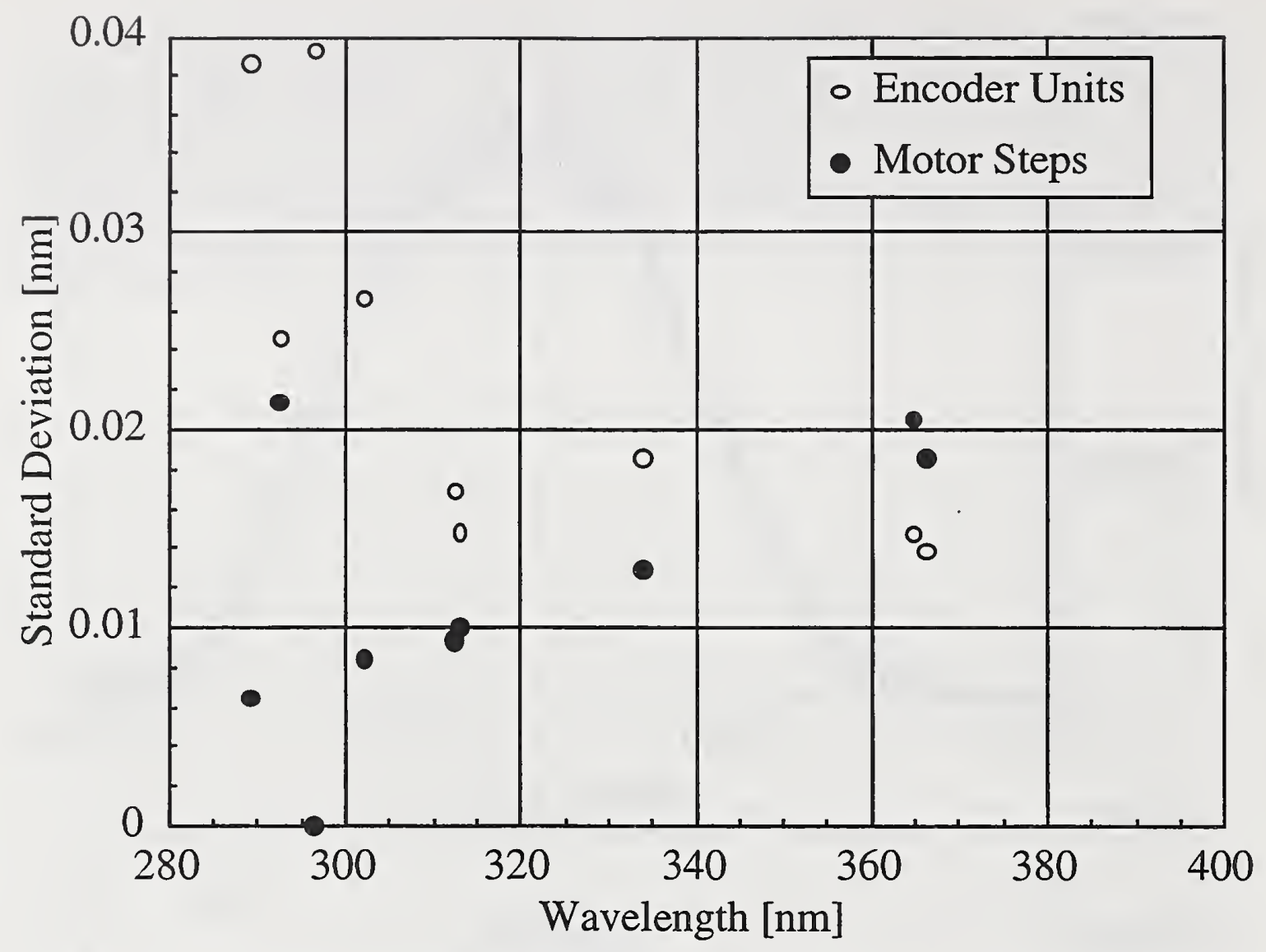

Figure 4.2. Standard deviations of the centroids from $\mathrm{Hg}$ emission lines as a function of wavelength in terms of the indicated diffraction grating angle units for Unit 901. 


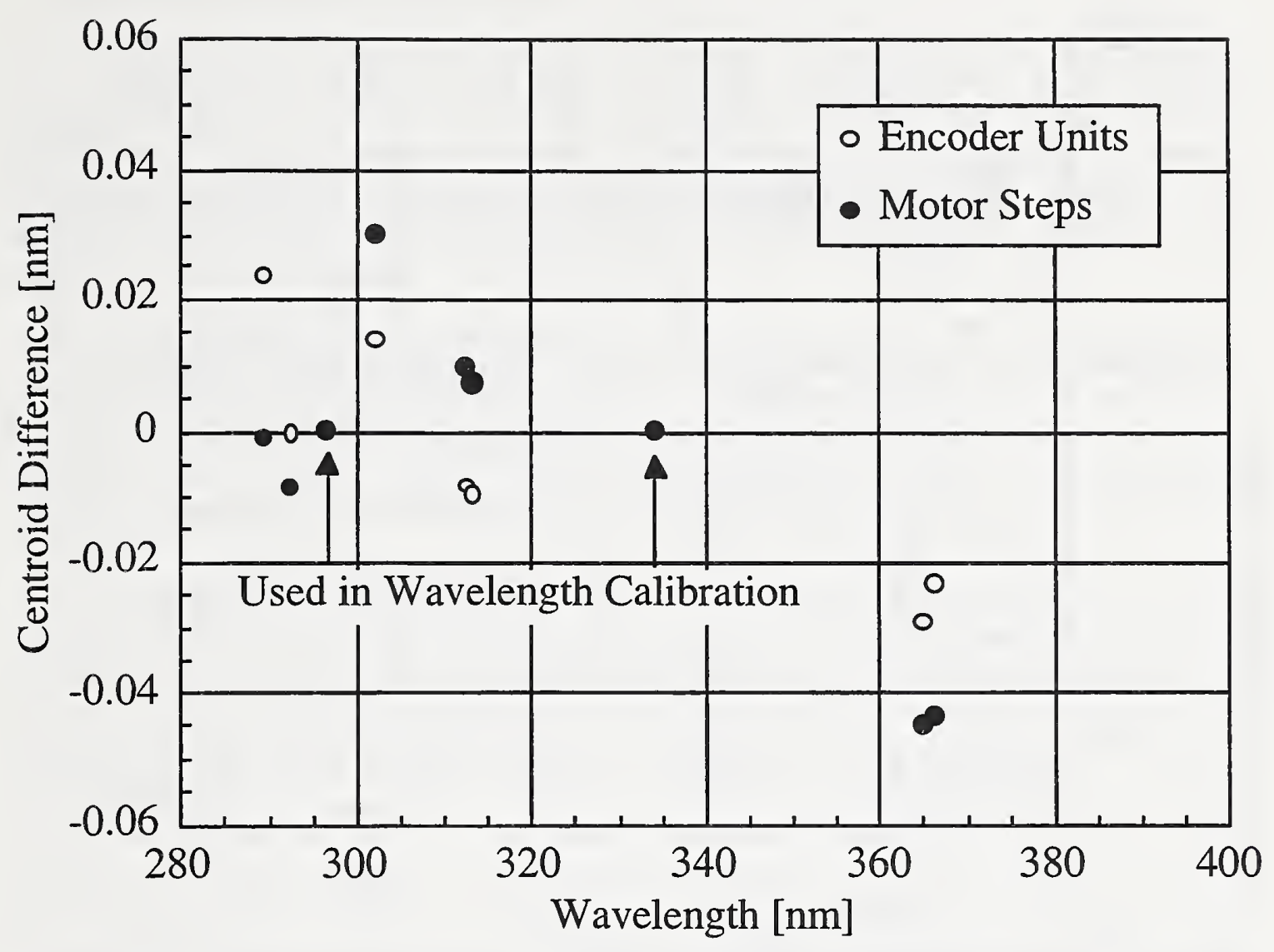

Figure 4.3. Average differences between centroids calculated from the wavelength calibration and the actual scans of $\mathrm{Hg}$ emission lines as a function of wavelength in terms of the indicated diffraction grating angle units for Unit 901 . The arrows indicate the wavelengths used to calibrate the wavelength of the monochromator. 


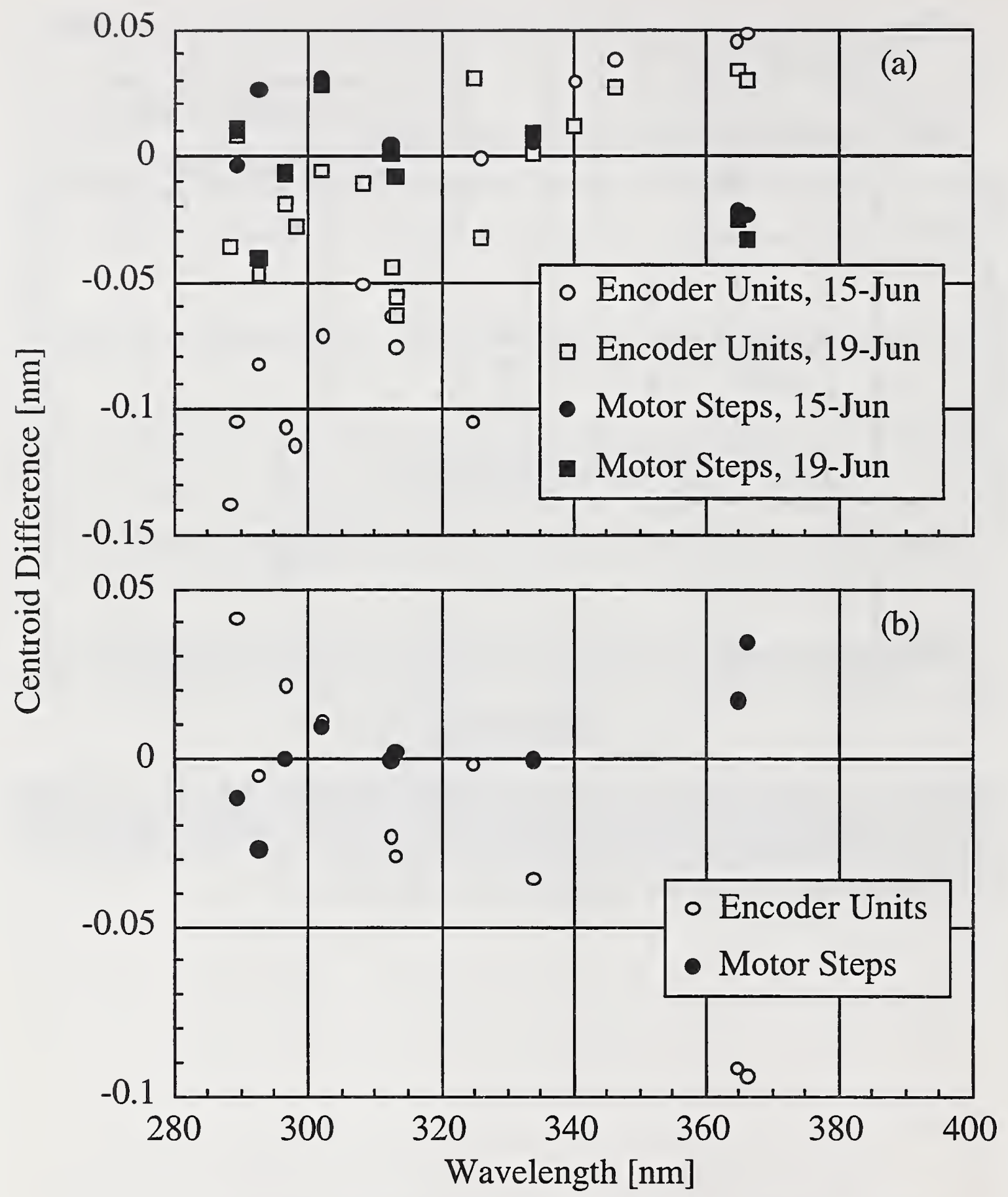

Figure 4.4. Differences between centroids calculated from wavelength calibrations and actual scans of emission lines as a function of wavelength for (a) Unit 901 and (b) Unit 902 at the Intercomparisons in terms of the indicated diffraction grating angle units and dates. 


\section{SLIT-SCATTERING FUNCTION}

Ideally, the slit-scattering function $z\left(\lambda-\lambda_{0}\right)$ is sharply peaked at $\lambda_{0}$ and triangular in shape about this peak as a function of wavelength. The full-width-at-half-maximum (FWHM) of the slit-scattering function is the bandwidth $\Delta \lambda$ of the instrument, which is important for comparing irradiances from instruments with different bandwidths and depends upon the widths of the entrance, center, and exit slits. In addition, the stray-light rejection of the monochromator was determined.

A HeCd laser operating at $325.029 \mathrm{~nm}$ with an output power of approximately $15 \mathrm{~mW}$ was used to obtain radiation at a specific wavelength as required for Eq. (2.4) to be valid. The output beam from the laser was directed onto the diffuser either directly or through a fiber optic cable. These measurements were done at both Boulder intercomparisons and at NIST on March 6, 1995 for Unit 901.

The slit-scattering function over a small wavelength range about $325 \mathrm{~nm}$ was obtained by performing spectral scans with wavelength intervals of approximately $0.01 \mathrm{~nm}$ in order to over-resolve the laser line. The wavelength of the monochromator was determined by the calibration in terms of encoder units. The signals were corrected for the dead time of the photon counting circuit and normalized by the peak signal. The peaknormalized signal, on a logarithmic scale, as a function of wavelength is shown in Fig. 5.1 for (a) Unit 901 and (b) Unit 902 . Note that, because the wavelength of the source is fixed while the wavelength of the monochromator is changed, Fig. 5.1 is a plot of $z\left(\lambda_{0}-\lambda\right)$ rather than $z\left(\lambda-\lambda_{0}\right)$. The slit-scattering function of each instrument has a Gaussian shape rather than the ideal triangle, and is slightly asymmetric about $325 \mathrm{~nm}$ for normalized signals greater than $10^{-4}$. This is likely due either to aberrations in the optics or to misalignment of the slits, or both. The asymmetry in Fig. 5.1(a) for smaller signals is discussed below.

There are additional peaks in Fig. 5.1(b) separated by $2 \mathrm{~nm}$ from the primary peak at $325 \mathrm{~nm}$. From experiments performed at both RSI and ASRC, the diffraction gratings are known to be defective. The additional peaks are possibly due to buckling of the coating on the diffraction grating. At the buckles, the angle of incidence and hence the angle of diffraction changes, resulting in peaks with reduced signal at wavelength settings of the monochromator other than $325 \mathrm{~nm}$.

Spectral scans with wavelength intervals of approximately $0.2 \mathrm{~nm}$ were performed to determine the slit-scattering function over the operating wavelength range of the instruments. The stray-light rejection is the most important piece of information obtained from such scans, as this determines the ability of the instrument to accurately measure solar irradiance at the shortest ultraviolet wavelengths. Because $z\left(\lambda_{0}-\lambda\right)$ is determined from these spectral scans, the stray-light rejection is the ratio of the smallest signals at the shortest wavelengths to the peak signal at the wavelength of the laser. Therefore, the largest peak signal possible is required to accurately determine the actual stray-light rejection. The peak signal was obtained by performing a spectral scan about $325 \mathrm{~nm}$ at a wavelength interval of $0.01 \mathrm{~nm}$ with the full power of the laser directed onto the diffuser of 
Unit 901. The measured signals were adjusted for the "roll-over" of the counts above $2^{20}$, which occurred several times, resulting in a peak signal of over $5 \times 10^{6}$ counts/s. The signals from the scan over the entire wavelength range were corrected for the dead time and dark count (obtained by signals with no light incident on the diffuser and equal to approximately 0.5 counts/s) of the photon counting circuit and normalized by the peak signal. The result is shown in Fig. 5.2, where the peak-normalized signal is plotted as a function of wavelength. The stray-light rejection of the monochromator is at least $10^{-8}$, and could be better than this value since the signals at the shortest wavelengths are nearly equal to the dark count.

The stray-light performance of the instrument when it is measuring solar irradiance depends not only on the stray-light rejection of the monochromator, but also on the spectral responsivity of the detector and the spectral irradiance of the source. As an example, a calculation of the approximate signal due to stray light when measuring solar irradiance is presented here. Separating Eq. (2.3) into components from wavelength regions within $2.5 \mathrm{~nm}$ of the monochromator wavelength and those regions that differ from the monochromator wavelength by more than $2.5 \mathrm{~nm}$, and converting the integrals to sums yields

$$
S\left(\lambda_{0}\right)=\sum_{i} E\left(\lambda_{i}\right) R\left(\lambda_{i}\right)\left(10^{-8}\right)(5 \mathrm{~nm})+E\left(\lambda_{0}\right) R\left(\lambda_{0}\right) \int z\left(\lambda-\lambda_{0}\right) d \lambda
$$

The first term on the right-hand-side of Eq. (5.1) is the contribution to the signal from stray light, while the second term is the contribution from the light at wavelengths within the bandwidth of the instrument. For the purpose of calculation, the values of $R\left(\lambda_{i}\right)$ and $R\left(\lambda_{0}\right) \int z\left(\lambda-\lambda_{0}\right) d \lambda$ were obtained from the spectral irradiance responsivity determined for Unit 901 on April 21, 1995. Since the PMT responds to light at wavelengths shorter than $600 \mathrm{~nm}$, while the irradiance response function was determined only for wavelengths shorter than $390 \mathrm{~nm}$, this function was assumed to be constant from $390 \mathrm{~nm}$ to $600 \mathrm{~nm}$. The solar irradiance $E\left(\lambda_{i}\right)$ was averaged over $5 \mathrm{~nm}$ intervals from measurements taken by Unit 901 at the second Intercomparison on June 21, 1995 at approximately solar noon. For wavelengths longer than $390 \mathrm{~nm}$, the solar irradiance was calculated from Planck's blackbody equation with a temperature of $5780 \mathrm{~K}$. The stray-light rejection of the monochromator was assumed to be $10^{-8}$, and the products of irradiance and responsivity were over $5 \mathrm{~nm}$ intervals. The fraction of signal due to stray light was calculated using Eq. (5.1), and is shown in Fig. 5.3 as a function of wavelength. Even with the high stray-light rejection of the monochromator, because of the rapid decrease in solar irradiance with decreasing wavelength at wavelengths shorter than $310 \mathrm{~nm}$, approximately half of the signal at $290 \mathrm{~nm}$ is due to stray light. This fraction decreases rapidly with increasing wavelength, and is only 0.0035 at $300 \mathrm{~nm}$ and approaches $10^{-5}$ at longer wavelengths. As a point of reference, the fraction at $290 \mathrm{~nm}$ when the source is a $1000 \mathrm{~W}$ FEL-type lamp is only 0.0029 . These results demonstrate the difficulty of determining accurately the solar irradiance in the UV-B region even with a monochromator with good stray-light rejection. 
The normalized signal in Fig. 5.2 is obviously enhanced for wavelengths longer than $325 \mathrm{~nm}$, which is presumably due to fluorescence in the diffuser. This fluorescence is also responsible for the asymmetry shown in Fig. 5.1(a). The fluorescence is visible to the naked eye but is not a problem with solar irradiance measurements since the irradiances at ultraviolet wavelengths are five orders of magnitude less than that from the laser. Thus, any signal from fluorescence is indistinguishable from the dark signal. The results for Unit 902 are similar to those shown in Fig. 5.2, although the stray-light rejection is not as well known because a less powerful laser was used. Since both monochromators are nominally identical, it is safe to assume that the stray-light rejection of Unit 902 is similar to that of Unit 901.

The bandwidth of each instrument was determined from the wavelength scans of the $\mathrm{HeCd}$ laser and of the singlet emission lines of the $\mathrm{Hg}$ and $\mathrm{Cd}$ lamps. This occurred for both instruments at the Intercomparisons and at NIST for Unit 901. The full-width-at-halfmaximum (FWHM) bandwidth is calculated by the difference in wavelength between the two signals that are half of the maximum signal for that line. The results are shown in Fig. 5.4 where the bandwidth is plotted as a function of wavelength for (a) Unit 901 and (b) Unit 902 . The bandwidth decreases with increasing wavelength, and is nominally $0.3 \mathrm{~nm}$ for both instruments. 


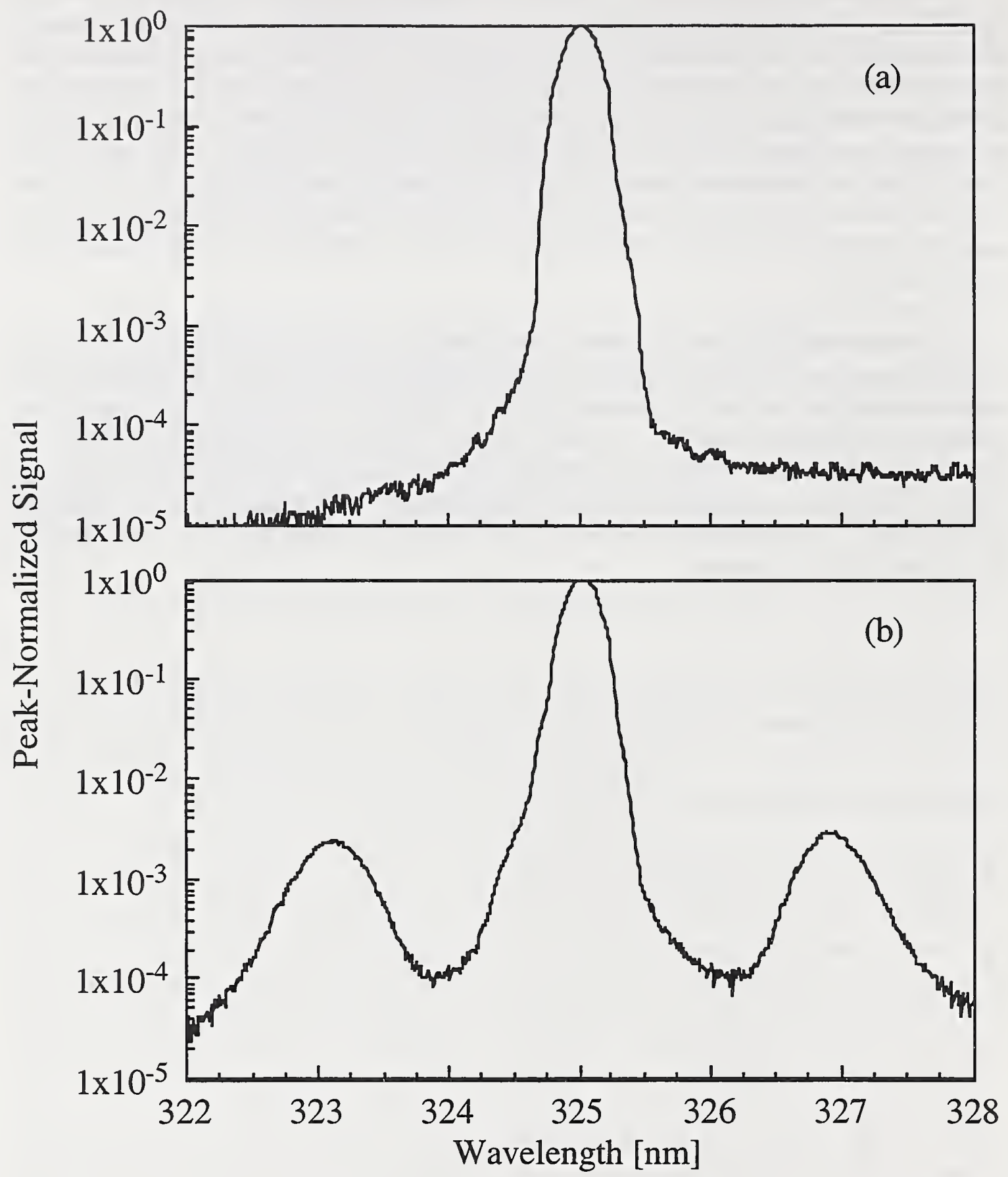

Figure 5.1. Peak-normalized signal as a function of wavelength of the monochromator from scans of a HeCd laser for (a) Unit 901 and (b) Unit 902. 


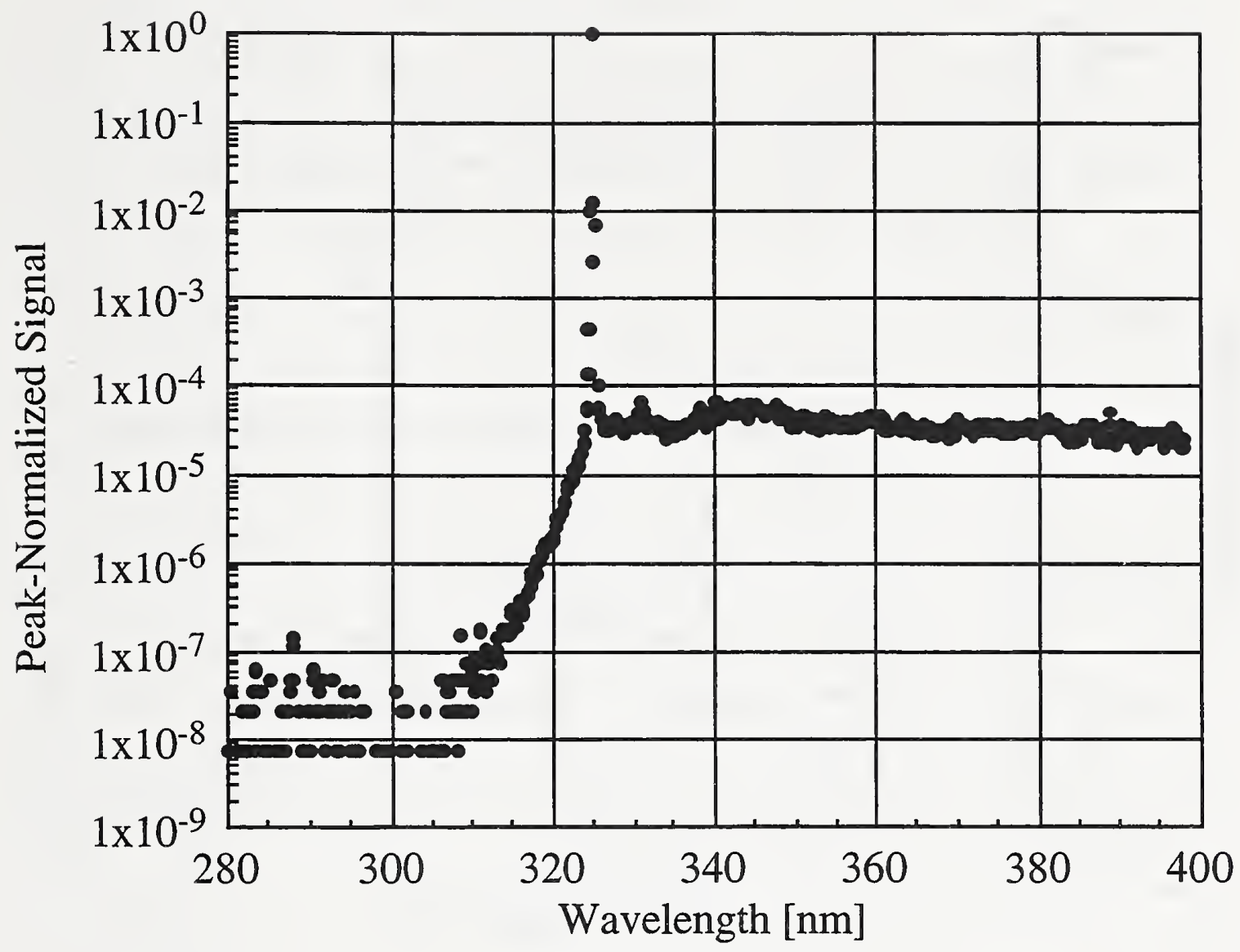

Figure 5.2. Peak-normalized signal as a function of wavelength of the monochromator from scans of a HeCd laser for Unit 901. 


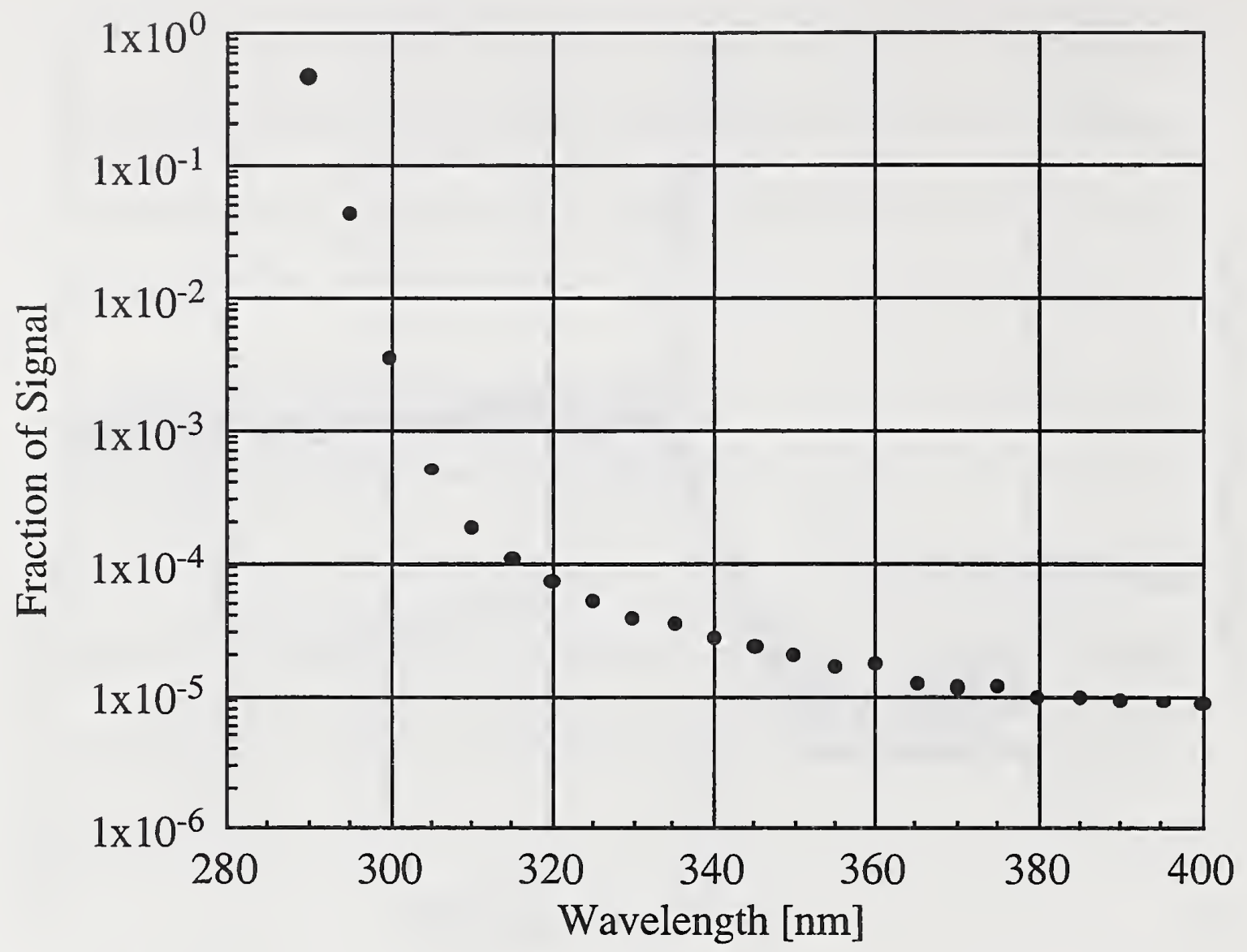

Figure 5.3. Fraction of total signal due to stray light as a function of wavelength for Unit 901 measuring solar irradiance. 


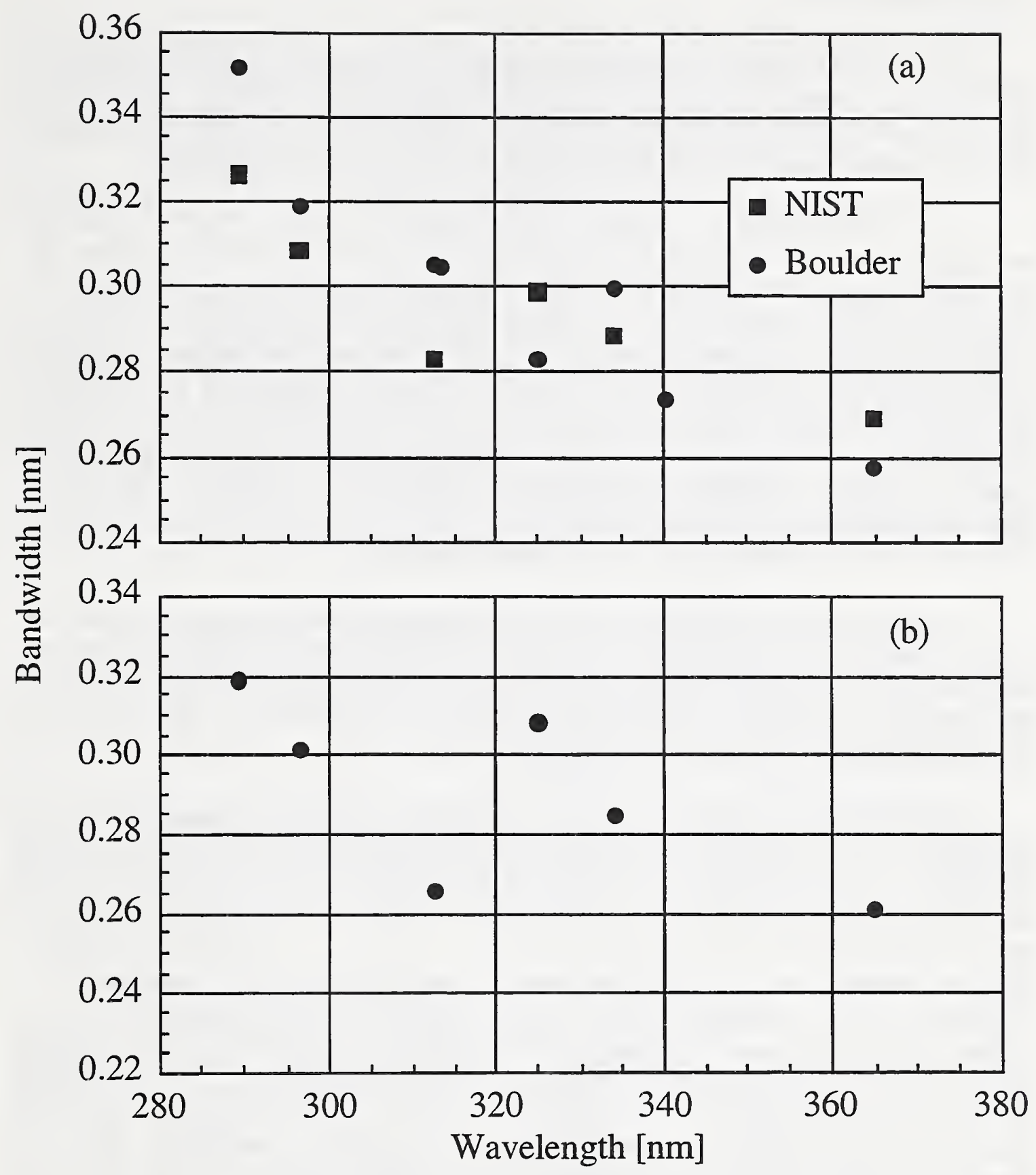

Figure 5.4. Bandwidth as a function of wavelength from scans of singlet emission lines for (a) Unit 901 at the indicated locations and (b) Unit 902. 


\section{RESPONSIVITY}

The spectral irradiance responsivity, hereafter simply termed the responsivity, of an instrument is a measure of its sensitivity to light at a given wavelength. In brief, the responsivity at each wavelength was determined from Eq. (2.5) by measuring the signal $S\left(\lambda_{0}\right)$ of the instrument and dividing by the irradiance $E_{\mathrm{s}}\left(\lambda_{0}\right)$ of a standard lamp.

The standard lamps were tungsten quartz-halogen FEL lamps with their irradiance determined by comparison with similar lamps calibrated at the NIST FASCAL facility [4]. Two different lamps were used, one at NIST and at the first Boulder intercomparison, designated OS-27, and another one at the second Boulder intercomparison, designated F-332. A lamp was positioned horizontally in a fixture with sufficient degrees of freedom to place it centered on, perpendicular to, and $50 \mathrm{~cm}$ from the diffuser. In the laboratory, this fixture was mounted on an optical table, while at the first Boulder intercomparison it was mounted on a tripod and at the second Boulder intercomparison it was mounted in a cylindrical housing. Alignment of the lamp with respect to the diffuser is described in detail elsewhere [5]. The current through the lamp was controlled and adjusted by a computer-controlled power supply, as described elsewhere [6].

A measurement of the responsivity of an instrument consisted of two spectral scans over the entire range. In the first scan, a shutter was placed halfway between the lamp and the diffuser to block the direct light from the lamp. Following the convention for solar measurements, this signal is termed the diffuse signal. The shutter was removed for the second scan, and the total signal was measured.

For the measurements at NIST, the wavelength interval was approximately $5 \mathrm{~nm}$, the integration time was $10 \mathrm{~s}$, and one diffuse and three total signals were recorded at each wavelength. The direct signal was simply the diffuse signal subtracted from the average of the total signal and was corrected for the dead time. The standard uncertainty in the signal due to random variations was the standard deviation of the mean of the three total signals. The direct signal was then interpolated with a natural cubic spline to wavelengths at multiples of $5 \mathrm{~nm}$. The responsivity of Unit 901 was determined several times with it oriented both vertically and horizontally. At the Boulder intercomparisons, the technique more closely followed the normal procedure for these instruments. The wavelength interval was approximately $0.2 \mathrm{~nm}$ for Unit 901 and $0.4 \mathrm{~nm}$ for Unit 902, the integration time was $2 \mathrm{~s}$, and one diffuse and one total signal was recorded at each wavelength. The direct signal was corrected for the dead time, and the standard uncertainty in the signal due to random variations was the square root of the number of photons counted. Again, the direct signal was interpolated with a natural cubic spline to wavelengths at multiples of $5 \mathrm{~nm}$, and the instruments were always oriented vertically.

The responsivity was obtained by dividing the direct signal by the lamp irradiance at each wavelength. Uncertainties in the determination of the responsivity enter from a variety of sources, and can be divided between those associated with the lamp and those associated with the instrument. The sources of uncertainty from the lamp are its calibrated irradiance, the alignment of the lamp with respect to the diffuser, and the current through the lamp. 
For the instrument, the sources of uncertainty are the random variations of the signal and the wavelength uncertainty. Of these sources, the ones that contribute significantly to the combined standard uncertainty are the irradiance of the lamp, the distance from the lamp to the diffuser, and the random variations of the signal. The expanded relative uncertainty in responsivity from the first source decreases smoothly from 0.012 at $280 \mathrm{~nm}$ to 0.010 at $400 \mathrm{~nm}$, is 0.008 from the second source, and is calculated as described in the preceding paragraph for the last source. Additional details about the uncertainties are given elsewhere [5].

The responsivity of Unit 901 as a function of wavelength from one determination at NIST is shown in Fig. 6.1. The expanded uncertainties in the values are the same size as the symbols. There is a marked decrease in responsivity at the shorter wavelengths, which is also observed in Unit 902 . Given a $250 \mathrm{~nm}$ blaze for the diffraction grating and the spectral response of the PMT, the responsivity is expected to have a maximum at approximately $280 \mathrm{~nm}$. The observation of a significantly reduced responsivity at the shortest wavelengths is another indication that the diffraction grating is defective. If the responsivity were the same at $290 \mathrm{~nm}$ as it is at $390 \mathrm{~nm}$, the fraction of the signal at $290 \mathrm{~nm}$ due to stray light when measuring the solar irradiance would be only 0.13 .

The responsivity of an instrument can change upon movement. Therefore, its translational stability is given by the ratio of the responsivity after the instrument is moved to the responsivity before it was moved. The results of this determination for Unit 901 at NIST are shown in Fig. 6.2 for the instrument in the (a) horizontal position and (b) vertical position, where the ratio of the responsivities is plotted as a function of wavelength. The dates on which the responsivities were determined are given in the legends, and the expanded uncertainties in the ratios are given by the vertical lines. The responsivity ratios differ markedly from one, indicating that the translational stability is poor. At several wavelengths in Fig. 6.2(b) the change is greater than $20 \%$, and in general the changes are on the order of $10 \%$.

The responsivity can also change when the instrument has not been moved, which is termed temporal stability. Two spectral scans of the total signal on the same day, one right after the other, were usually performed with Unit 901 at NIST. The ratio of the responsivity determined from the second scan to that from the first scan as a function of wavelength is shown in Fig. 6.3 for the instrument in the (a) horizontal position and (b) vertical position. The legends and vertical bars have the same meanings as in the previous figure. The ratios are equal to one within the expanded uncertainties, and the uncertainty increases at shorter wavelengths because both the irradiance and the responsivity are lower than at longer wavelengths.

The translational stability of Unit 901 was determined at the Boulder intercomparison. The responsivity ratios of the instrument after movements from NIST to the Boulder intercomparison site and from the indoor laboratory to the outdoor pad are plotted in Fig. 6.4(a) as a function of wavelength. The responsivity at the Boulder intercomparison was only about $40 \%$ of what it had been at NIST. This was due to the different PMT and photon counting circuit between the two determinations since the ratio is 
nearly independent of wavelength. On the other hand, the stability upon moving from indoors to outdoors was poor, with the responsivity increasing by nearly $60 \%$ at the shortest wavelengths. The outdoor temporal stability of both instruments was determined at the Boulder intercomparisons. The results are shown in Fig. 6.4(b), where the responsivity ratios for both instruments are plotted as a function of wavelength. The responsivity of Unit 901 definitely increased by approximately $4 \%$ at wavelengths longer than $340 \mathrm{~nm}$, while any increase at shorter wavelengths is less than $10 \%$. For Unit 902, the responsivity decreased dramatically to approximately $60 \%$ of its previous value.

The instability of the responsivity is very troublesome, as it demonstrates that the instruments are unsuitable for long-term, accurate measurements of solar ultraviolet irradiance. A likely cause for the instability is mechanical flexure of the instrument, particularly of the optical components. If the optics are shifted every time the instrument is moved, then the optical throughput will change, resulting in a different responsivity. This would account for the poor translational stability shown in Figs. 6.2 and 6.4(a), whereas the temporal stability shown in Fig. 6.3 is adequate. However, the temporal instabilities outdoors, shown in Fig. 6.4(b), particularly since they are over periods of only a few days, indicates that there is also an environmental effect on the responsivity. 


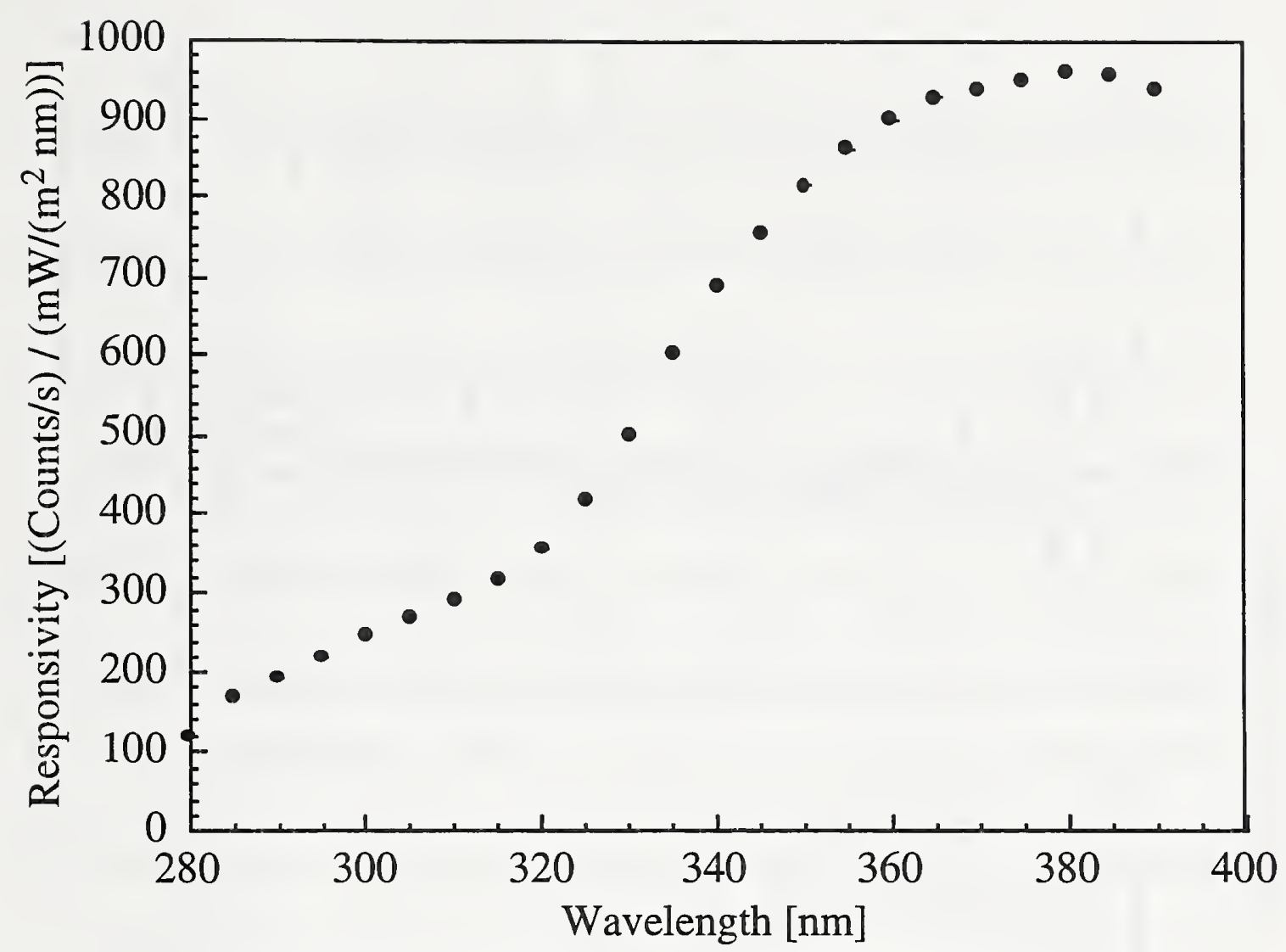

Figure 6.1. Responsivity as a function of wavelength for Unit 901 oriented vertically at NIST. 


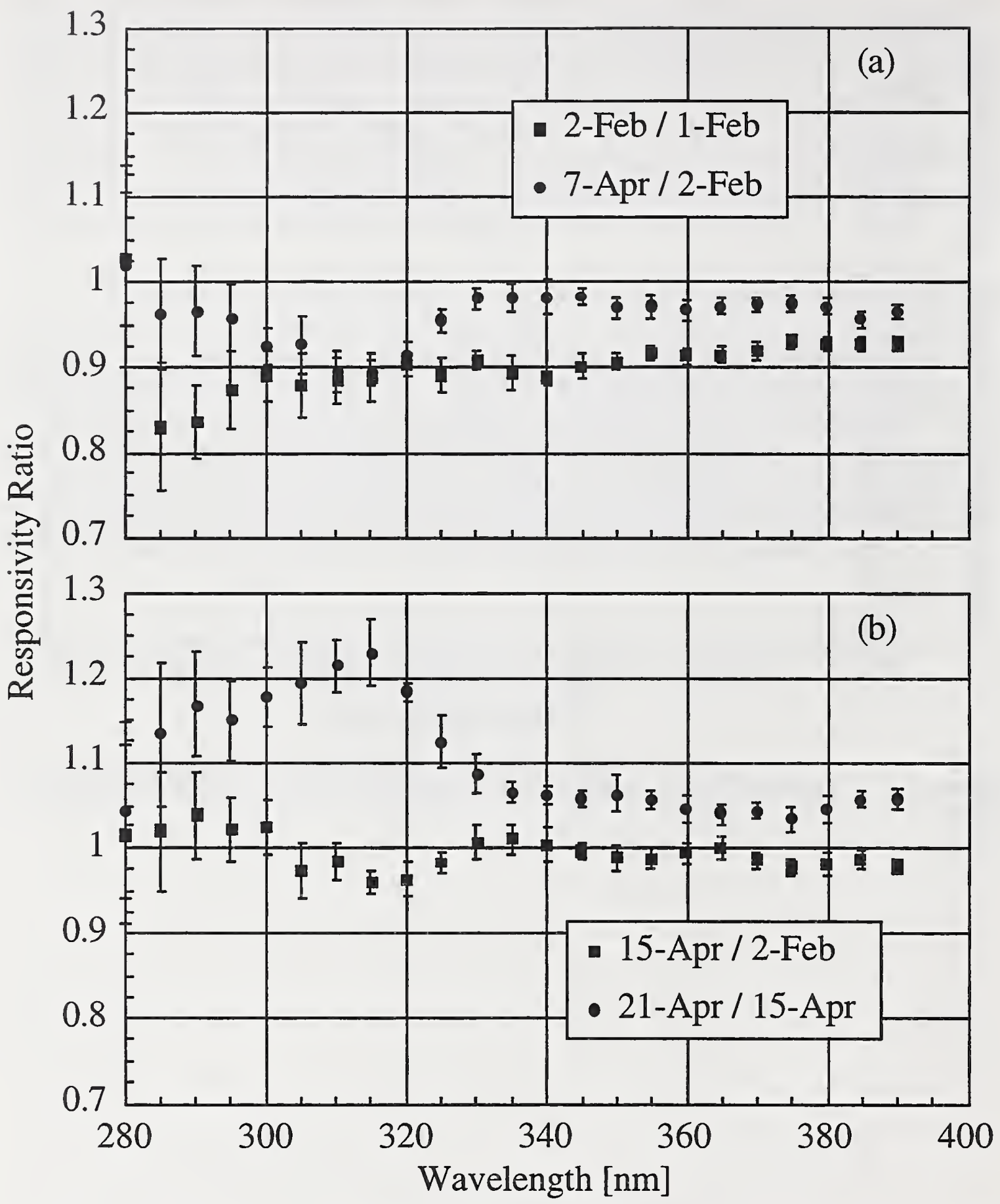

Figure 6.2. The translational stability of Unit 901 at NIST, given by the ratio of the responsivity after movement to the responsivity before movement as a function of wavelength with the instrument oriented (a) horizontally and (b) vertically on the indicated dates. The expanded uncertainties (coverage factor $k=2$ ) in the ratios are given by the vertical bars. 


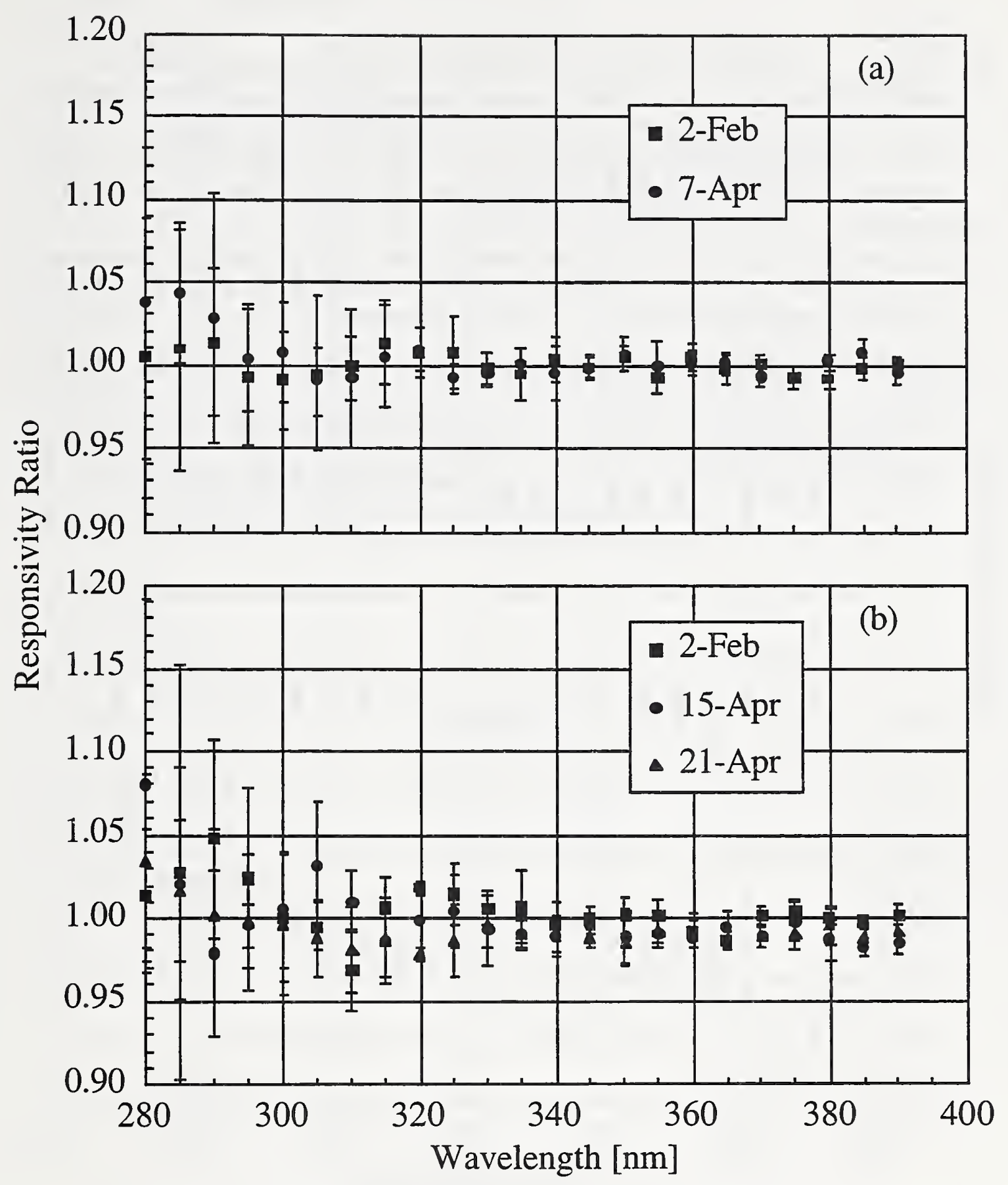

Figure 6.3. The temporal stability of Unit 901 at NIST, given by the ratio of two successive responsivities as a function of wavelength with the instrument oriented (a) horizontally and (b) vertically on the indicated dates. The expanded uncertainties (coverage factor $k=2$ ) in the ratios are given by the vertical bars. 


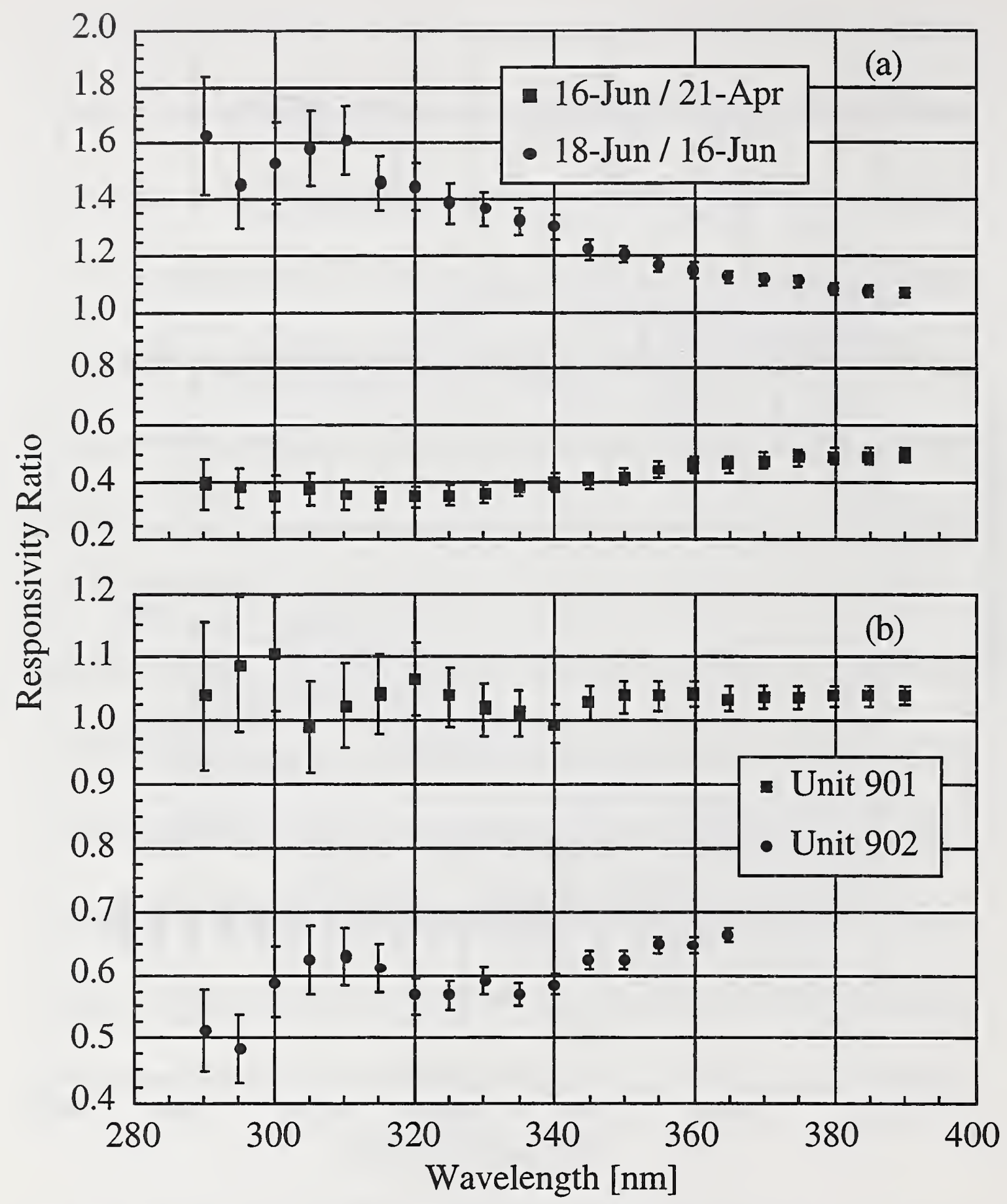

Figure 6.4. (a) Translational stability of Unit 901 at the Intercomparison, given by the ratio of the responsivity after movement to the responsivity before movement as a function of wavelength on the indicated dates. (b) Temporal stability of Units 901 and 902 at the Intercomparisons, given by the ratio of responsivities determined on two days while the instruments were outdoors. The expanded uncertainties (coverage factor $k=2$ ) in the ratios are given by the vertical bars. 


\section{SIGNAL UNCERTAINTY}

An assumption with photon counting circuits is that the uncertainty in the signal due to random variations is given by Poisson statistics, namely that the standard uncertainty in the number of photons detected is given by the square root of that number. To test the validity of this assumption, a standard lamp was used to irradiate the diffuser of Unit 901 at NIST, and signals were recorded at four different fixed wavelengths for 2 min with $1 \mathrm{~s}$, $5 \mathrm{~s}$, and $10 \mathrm{~s}$ integration times. The signal was converted to photons by multiplying by five, and the standard deviation of the values and the square root of the average were calculated. The ratio of the standard deviation to the square root is plotted in Fig. 7.1 as a function of the signal, and averages 2.5. Thus, the standard uncertainty of the signal is significantly greater than that expected from Poisson statistics, regardless of the signal, showing that there are additional sources of random variation in the signal. The situation improved when similar measurements were performed at the Boulder intercomparison, also shown in Fig. 7.1, where the average of the ratio is 1.2, close to the expected value of 1.0. The improvement in the uncertainty of the signal is likely due to the different PMT and photon counting circuits between the two determinations. 


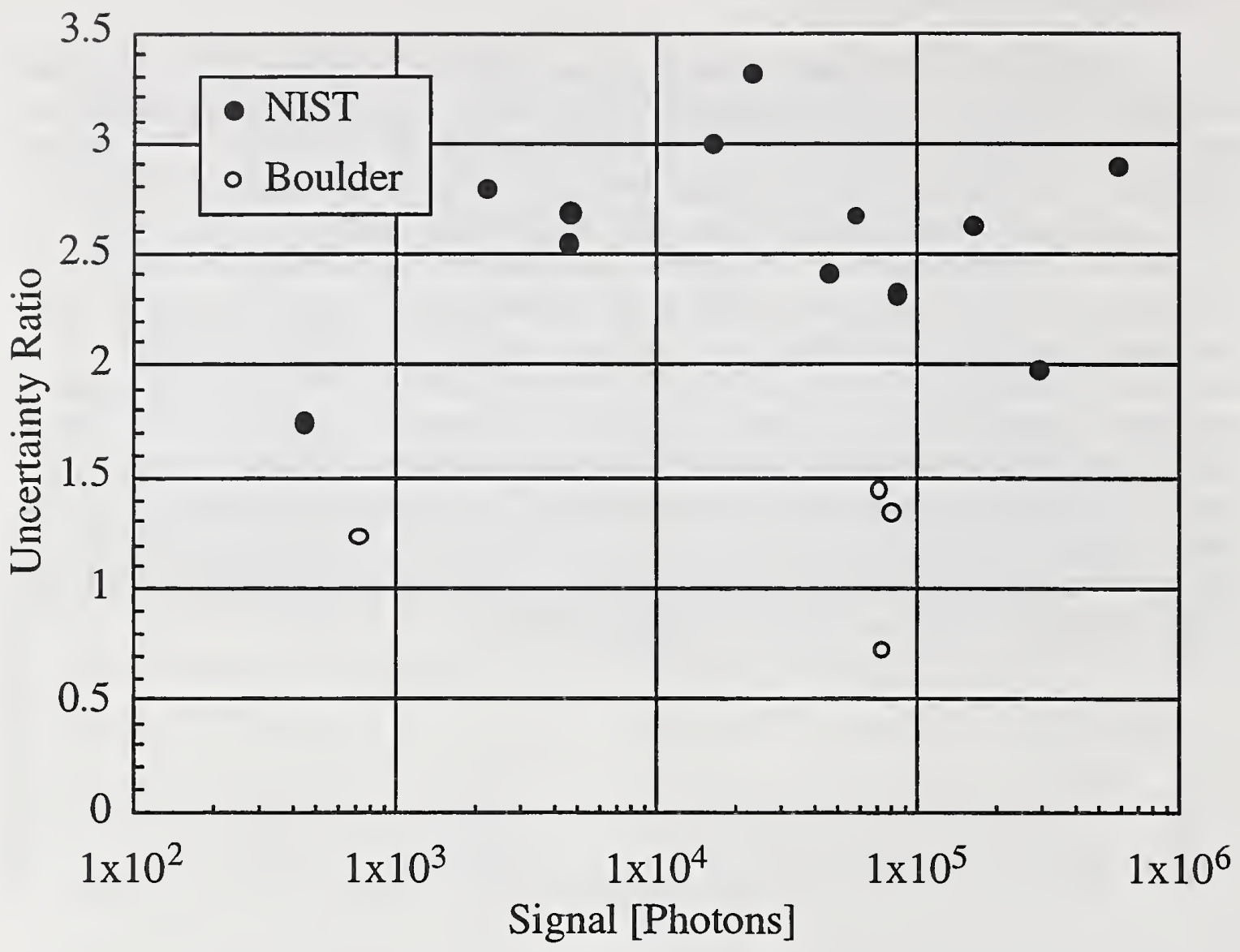

Figure 7.1. The ratio of the uncertainty of the signal determined from the standard deviation to that determined from the square root of the signal as a function of signal for Unit 901 at the indicated locations. 


\section{CONCLUSIONS}

From the characterizations detailed in this report, several conclusions emerge about the performance of these instruments and their suitability for long-term, accurate monitoring of solar ultraviolet irradiance. The linearity of the detector system is adequate, especially since corrections for the dead time of the photon counting circuit can easily be made to the signals. The wavelength repeatability and uncertainty are more problematic. Because of the problems in the mechanical coupling between the nut on the lead screw and the encoder unit, calibrating the wavelength of the monochromator in terms of motor steps rather than encoder units is preferred. Even so, the wavelength repeatability is only $0.02 \mathrm{~nm}$, while the uncertainty of $0.04 \mathrm{~nm}$ is worse, and can be even greater for individual spectral scans.

The bandwidth is $0.3 \mathrm{~nm}$ for both instruments, although the shape of the slitscattering function indicates some slight optical aberrations or slit misalignment. The straylight rejection of $10^{-8}$ is satisfactory for a double monochromator, and fluorescence in the diffuser will not be a problem for solar measurements. However, the defective diffraction grating is a major problem. This causes the additional peaks in the slit-scattering function of Unit 901 and, more importantly, the decrease in responsivity at shorter wavelengths. This significantly compromises the ability of the instrument to accurately measure the solar irradiance at these wavelengths.

The instability of the instrument responsivity, both translational and temporal, is another major problem, as it implies that the instruments are not stable enough to detect long-term, or even short-term, changes in solar ultraviolet irradiance. A responsivity determined in the laboratory is not the same as that determined in the field, and the responsivity in the field is not stable. This instability is probably caused by both mechanical and environmental effects. Any detected changes in solar irradiance could rightly be attributed to changes in the instrument and not to changes in the transmittance of the atmosphere. Finally, the uncertainty of the signals was greater than expected, although this was decreased with a different PMT and photon counting circuit. 


\section{REFERENCES}

[1] H. J. Kostkowski and F. E. Nicodemus, An Introduction to the Measurement Equation, Chapter 5 of Self-Study Manual on Optical Radiation Measurements: Part I - Concepts, Natl. Bur. Stand. Tech. Note 910-2 (1978).

[2] B. N. Taylor and C. E. Kuyatt, Guidelines for Evaluating and Expressing the Uncertainty of NIST Measurement Results, NIST Technical Note 1297 (1993).

[3] Ambler Thompson and H.-M. Chen, "Beamcon III, a Linearity Measurement Instrument for Optical Detectors," J. Res. Natl. Inst. Stand. Technol., 99, 751 (1994).

[4] J. H. Walker, R. D. Saunders, J. K. Jackson, and D. A. McSparron, Spectral Irradiance Calibrations, NBS Special Publication SP 250-20 (1987).

[5] E. A. Early and Ambler Thompson, "Irradiance of Horizontal Quartz-Halogen Standard Lamps," J. Res. Natl. Inst. Stand. Technol., 101, 141 (1996).

[6] J. H. Walker and Ambler Thompson, "Improved Automated Current Control for Standard Lamps," J. Res. Natl. Inst. Stand. Technol., 99, 255 (1994). 

